\title{
La generatividad a través de la cultura. Una revisión sistemática
}

\section{Generativity through culture. A systematic review}

\author{
Álvaro Benito-Ballesteros \\ Universidad Complutense de Madrid, Madrid, España \\ alvbenit@ucm.es \\ Iria de la Osa Subtil \\ Universidad Complutense de Madrid, Madrid, España \\ iridelao@ucm.es
}

\begin{abstract}
Resumen: Esta revisión sistemática pretende resolver la controversia que existe en la relación entre generatividad y cultura, para así clarificar el modo de estudio generativo desde una perspectiva cultural en futuras investigaciones empíricas. Los artículos incluidos en la revisión, publicados entre 1990 y 2019, debían ser artículos científicos revisados por pares con contenido explícito acerca de ambos términos. En la búsqueda se utilizaron cinco bases de datos combinando repositorios multidisciplinares con bases especializadas, obteniendo un total de treinta artículos elegibles para esta revisión $(n=30)$. El análisis de los artículos se articula en cuatro categorías que aportan información acerca de diferentes dimensiones de la relación entre ambos conceptos: los límites de la generatividad cultural, comparaciones transculturales de la estructura de la generatividad, efectos e interpretaciones culturales de la generatividad y evidencias culturales de su aplicación y utilidad potencial. En conclusión, los aspectos más psicológicos que estructuran la generatividad son comunes interculturalmente, sin embargo, los motivos, la concepción y la interpretación del comportamiento generativo parecen variar intensamente de un tipo de cultura a otra.
\end{abstract}

Palabras clave: generatividad, cultura, factores socioculturales, diferencias transculturales.

Abstract: This systematic review aims to resolve the controversy that exists in the relationship between generativity and culture, in order to clarify the approach of generative study from a cultural perspective in future empirical research. The articles included in the review, published between 1990 and 2019, should be 
peer-reviewed scientific articles with explicit content about both terms. In the search, five databases were used combining multidisciplinary repositories with specialized databases, obtaining a total of thirty articles eligible for this review $(n=30)$. The analysis of the articles is divided into four categories that provide information about different dimensions of the relationship between both concepts: the limits of cultural generativity, cross-cultural comparisons of the structure of generativity, effects and cultural interpretations of generativity, and cultural evidences of its application and potential usefulness. In conclusion, the most psychological aspects that structure generativity are common interculturally, however, the motives, conception and interpretation of generative behavior seem to vary intensely from one type of culture to another.

Keywords: generativity, culture, sociocultural factors, cross-cultural differences. 


\section{INTRODUCCIÓN}

Ciertamente, el concepto de generatividad nunca ha sido fácil de definir, y aún más controversia ha provocado el establecimiento de sus límites conceptuales por parte de algunos autores. De igual manera, el conocimiento que tenemos de la generatividad y su relación con la cultura se basa hoy en día en las aportaciones de los escasos estudios publicados que han perseguido objetivos muy diversos. Estos dos aspectos, generatividad y cultura, son los conceptos centrales de esta revisión sistemática, así como la relación que existe entre ambos.

Con el ánimo de facilitar al lector la compresión de esta revisión, se proporcionan para comenzar las definiciones de generatividad más utilizadas empleadas por los autores que más han aportado en el estudio generativo. Erikson (1963) concebía la generatividad como el interés (concern) por desarrollar y guiar a la siguiente generación. Dos décadas después Kotre (1984) redefinió el concepto como una especie de deseo de invertir la propia sustancia (substance) en formas de vida y trabajo que sobrevivirán a uno mismo. Más tarde, los autores a su vez fueron incluyendo referencias directas a las futuras generaciones: "En la generatividad, el adulto nutre, enseña, lidera y promociona la siguiente generación mientras genera productos vitales y resultados que benefician el sistema social y promocionan su continuidad de una generación a la siguiente" (McAdams y De St. Aubin, 1992: 1003); o: "El interés (concern) y compromiso de promocionar el desarrollo y bienestar de futuras generaciones" (McAdams, 2006: 81).

Erik Erikson fue el primero que definió el concepto de generatividad en 1963. Para Erikson, la generatividad se situaba en la séptima de las ocho etapas del desarrollo psicosocial de las personas (Erikson, 1963). En esta etapa del desarrollo, el adulto se debate entre el comportamiento generativo y el estancamiento (stagnation). Más tarde, el propio Erikson (1982) apuntó que el compartimiento generativo continuaba y podía observarse también en la vejez, no siendo exclusivo de la adultez, y lo denominó grand-generativity. Dos décadas después de la descripción inicial del concepto por parte de Erikson, Kotre $(1984,1996)$ sugirió que la generatividad probablemente no fuera una etapa vital, sino más bien un impulso que surge a lo largo de la vida. En concordancia con Erikson, McAdams y De St. Aubin presentaron el concepto de generatividad como un constructo psicosocial multifacético compuesto por siete elementos empíricos: deseo interior (inner desire), demanda cultural (cultural demand), interés por la generatividad (concern), pensamiento generativo (belief), compromiso generativo (commitment), acción (action) y narración (narration). 
Lo más interesante para esta revisión es que de los trabajos citados anteriormente que representan la estructura central del concepto de generatividad y su desarrollo a lo largo de la historia todos ellos hablan de una manera u otra de que la cultura podría tener un papel fundamental en el comportamiento generativo. El modelo de Erikson hace énfasis en la influencia que el ámbito social, cultural y ambiental tiene sobre el desarrollo psicosocial. Kotre (1984) señala la importancia de la cultura en la expresión generativa y define cuatro tipos de generatividad, entre los que incluye la generatividad cultural, que hace referencia a que el individuo no solo actúa de profesor, sino también de mentor, ayudando a otras personas a desarrollar el conocimiento de uno mismo. McAdams y De St. Aubin (1992), por su parte, no solo describen el concepto de demanda cultural, descrito como una de las fuentes de motivación externa de la generatividad, sino que también expresan que la cultura ejerce una influencia en la expresión generativa, ya que la sociedad en sí implementa la responsabilidad por las siguientes generaciones mediante los roles de padres, profesores o líderes.

Desde la publicación de los trabajos anteriores, han sido muchos autores los que han reivindicado el mayor estudio del papel de la cultura en el comportamiento generativo. Hofer et al., en 2008, ya acusaban la falta de estudios generativos transculturales. Más específicamente, Alexander, Rubinstein, Goodman y Luborsky sostenían que el dominio psicológico está inmerso en el marco cultural y que, por ello, el análisis del concepto de la generatividad no puede ser entendido a menos que se sitúe en contexto cultural particular. Argumentaban que la urgencia de la acción generativa resultaba de una matriz de pensamientos sobre la muerte, uno mismo, la nutrición de las siguientes generaciones (nurturance), el ámbito económico y el género, todos ellos pensamientos específicos con base en la cultura (1991: 421). En los últimos años, investigadores como Cheng (2009) o De Medeiros, Rubinstein y Ermoshkina (2013) han reivindicado el estudio del ámbito social y cultural como contexto y sistema simbólico en el que tiene lugar el comportamiento generativo.

Esta revisión tiene el objetivo de proporcionar y establecer una visión integradora de la relación que existe entre la generatividad y la cultura, un objeto de estudio que ha cobrado importancia en las últimas décadas (Figura 1). Pretende aclarar si en futuras investigaciones la cultura debe ser el contexto en el que se produce el comportamiento generativo, o una variable que afecta a la misma concepción del concepto. Reunir y reconciliar lo que sabemos acerca de la relación de ambos conceptos es imperativo para implementar una base sólida y una guía para las futuras investigaciones teóricas y empíricas de la generatividad y la cultura en las ciencias sociales. 


\section{FIGURA 1}

Publicaciones científicas acerca de generatividad y cultura

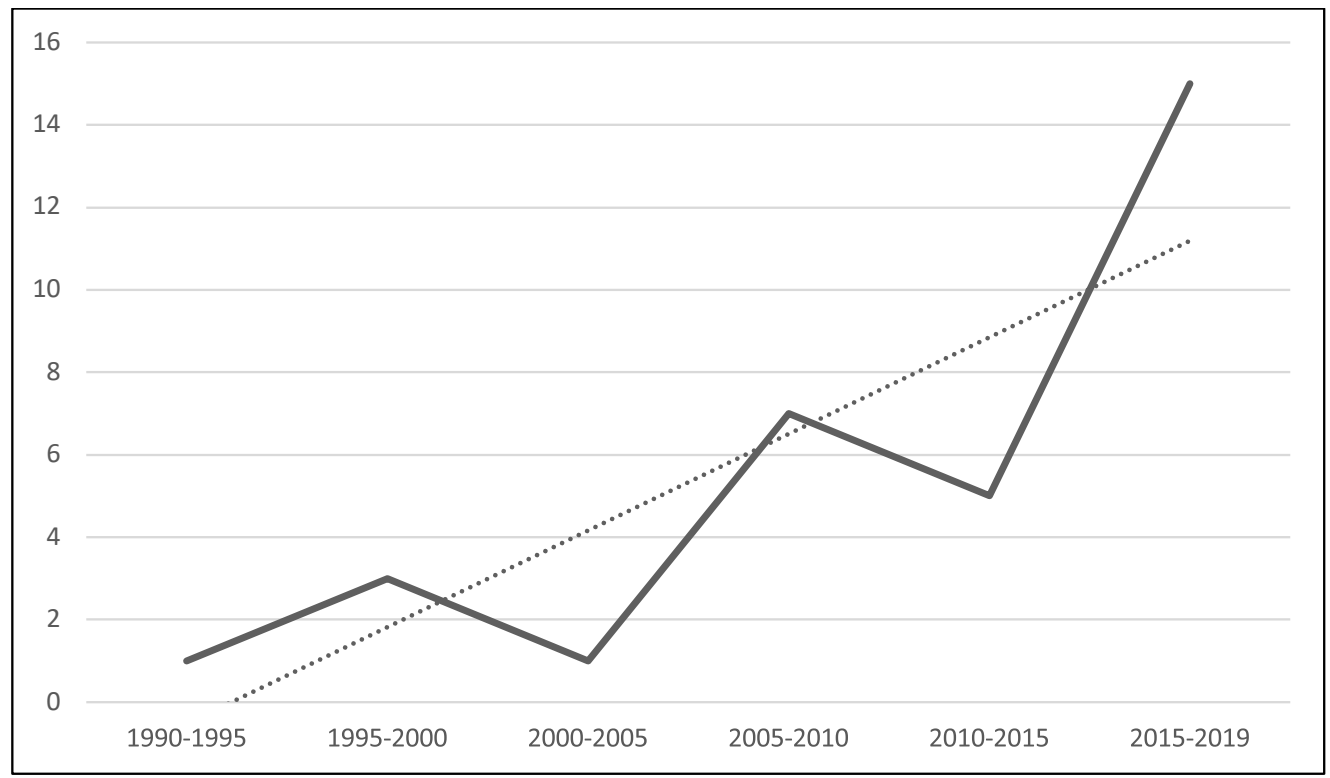

\section{MÉTODO}

Debido al razonamiento expuesto anteriormente y al estado del conocimiento disponible sobre la relación entre generatividad y cultura, esta revisión sistemática comenzó con un diseño de búsqueda en torno a estos dos conceptos: "cultura" (culture) y "generatividad" (generativity). Además, con el objetivo de maximizar la exhaustividad de la búsqueda, se incluyeron a su vez "diferencias transculturales" (cross cultural differences) y "factores socioculturales" (sociocultural factors), siendo necesaria para la recuperación de publicaciones la aparición del término generatividad y alguno de los tres restantes referidos a cultura (cultura, diferencias transculturales o factores socioculturales). Para alcanzar la máxima representatividad de los términos de búsqueda se combinó el uso de repositorios multidisciplinares con bases de datos especializadas en psicología. Las bases de datos utilizadas fueron Scopus, Web of Science, PsycInfo, Dialnet y Psicodoc (en el caso de esta última, al presentar únicamente trabajos en español se adaptó la estrategia de búsqueda estableciendo generatividad y cultura como términos clave). Con el ánimo de captar la evolución del estudio de la relación entre generatividad 
y cultura desde su origen se incluyeron trabajos publicados desde 1990 hasta 2019 (Figura 1).

La búsqueda quedó restringida a aquellos artículos científicos, revisados por pares, y con contenido explícito acerca de generatividad y cultura. Se excluyeron de la búsqueda aquellos artículos de disertación teórica, sin contenido relevante para la revisión o con el grupo cultural sin suficiente especificación. Tras el proceso de búsqueda y depurado (Figura 2) se obtuvieron treinta referencias susceptibles de ser incluidas en esta revisión $(n=30)$.

FIGURA 2

Estrategia de búsqueda y depurado

162 referencias totales

Web of Science: 45; Scopus: 45; PsycINFO: 69; Dialnet: 2; Psicodoc: 1.

Análisis y exclusión de duplicados: 110 referencias elegibles

Revisión de títulos y resúmenes: 42 referencias elegibles.

64 referencias sin contenido explícito de generatividad y cultura.

4 referencias de artículo de disertación o revisión teórica.

Revisión de acceso abierto y público al documento y texto completo: 30 referencias incluidas en la revisión.

\section{RESULTADOS}

Tras el análisis exhaustivo y la recopilación de toda la información relevante presente en los artículos que conforman esta revisión, se pasó a categorizar la información en 
cuatro epígrafes que serán presentados a continuación. El orden de los epígrafes responde al análisis de los conceptos de generatividad y cultura desde sus límites conceptuales y similitudes a través de diferentes culturas, hasta las diferencias culturales más drásticas y visibles, finalizando con las aplicaciones potenciales que ha demostrado tener el comportamiento generativo en distintos ámbitos relacionados con la cultura.

\section{Definiendo los límites de la generatividad cultural}

El trabajo de Kotre en 1984 no solo supuso avances teóricos muy notables en la concepción del comportamiento generativo, sino que definió lo que se conoce como generatividad cultural. Kotre describe este tipo de generatividad como aquella que proporciona un mecanismo que permite la perpetuación del conocimiento, sabiduría y valores culturales más allá del aquí y ahora. Una década después, Manheimer (1995) volvía a establecer los límites de la generatividad cultural asignándole la transmisión intergeneracional de tradiciones, valores y conocimiento de una cultura. Manheimer sostenía que para que un sujeto fuera capaz de transmitir generativamente una cultura, debería sentirse necesariamente partícipe de la misma. Este puede ser el primer indicio que deja entrever que una cultura distinta podría dar lugar a comportamientos generativos relativamente diversos, ya que las personas son partícipes de la cultura a la que pertenecen y no de las demás.

Otros autores (Lewis y Allen, 2017) defendieron que la generatividad cultural podía reducirse a la protección-transmisión de la cultura familiar y comunitaria entre generaciones. El lenguaje, las tácticas de supervivencia o el estilo de pensamiento irían inmersos en el concepto de cultura. Ciertamente, es lógico etiquetar de esta manera a la transmisión de valores y normas culturales, sin embargo, resultaría ingenuo pensar en la cultura como un conjunto de tradiciones, símbolos y valores que es simplemente transmitido y no afecta en nuestra conducta. Kane (1996) señaló que la expresión de la generatividad cultural se vería influida por aquellos que formaban parte de esa cultura y por aquellos que la formarán en el futuro, lo cual implica que la propia expresión cultural se vería influida ya por la cultura. Haciendo los límites aún más difusos, parece que el rango de comportamientos que pueden definirse como generativos culturalmente era más amplio que el establecido en un principio. Por ejemplo, autores como Peterson y Duncan (1999) señalaron que demostrar interés por la política contemporánea también podría ser considerado una expresión cultural generativa.

Kotre distingue entre cuatro tipos de generatividad: biológica, parental, técnica y cultural. Para este autor la generatividad cultural "transformaba los defectos en virtudes 
y validaba el yo dándole una desembocadura generativa" (1996: 260). Sin embargo, en estudios como el de Warburton, McLaughlin y Pinsker (2006), eran los propios participantes los que hablaban de la importancia de transmitir conocimientos técnicos y prácticos como símbolos de su cultura. Este ejemplo ilustra la dificultad que tiene la concepción del comportamiento generativo sin la cultura. ¿El papel de la cultura se limita entonces a la transmisión de valores y tradiciones? Es complicado asumir que la cultura dé lugar a un tipo de generatividad diferente del resto de comportamientos generativos. Más allá de la transmisión intergeneracional de valores y tradiciones culturales, cabe la duda en el establecimiento de un límite para el impacto que la cultura puede ejercer en el comportamiento generativo. Como se expondrá en los siguientes apartados, la cultura engloba al comportamiento generativo, afectando a su concepción, interpretación y expresión.

\section{Comparaciones transculturales de la estructura de la generatividad}

Una de las incógnitas que emergían repetidamente en el ámbito de la generatividad transcultural era si su estructura podía variar en función de la cultura. Como se expone en el apartado anterior, delimitar la cultura no parece una solución ni mucho menos definitiva. En ese sentido, examinar la estructura de la generatividad a través de diferentes culturas resulta un buen comienzo para estudiar la influencia de la cultura. Como se ha comentado anteriormente, los autores que contaron con más apoyo en la articulación conceptual de la generatividad fueron McAdams y De St. Aubin. La generatividad se operativizó en un modelo (Figura 3: McAdams, Hart y Maruna, 1998) conformado por siete elementos empíricos: deseo interior (inner desire), demanda cultural (cultural demand), interés por la generatividad (concern), pensamiento generativo o creencia (belief), compromiso generativo (commitment), acción (action) y narración (narration).

Este modelo de la generatividad y sus transformaciones (McAdams y De St. Aubin, 1992; McAdams, Hart y Maruna, 1998) fueron puestos a prueba en diferentes culturas que diferían entre ellas significativamente, como Camerún, Costa Rica, Alemania, China, República Checa... (Hofer et al., 2014; Hofer, Chasiotis, Busch, Kaetner y Campos, 2008). El ajuste del modelo fue notablemente óptimo independientemente de la cultura de la muestra. En otro estudio (Robinson et al., 2016) acerca de los procesos básicos y factores que influían en la admiración de personajes públicos, la generatividad era uno de los factores más admirados. Este hallazgo fue común para muestras procedentes de países como Inglaterra, Rusia, Irán o China. Otros efectos relacionados con la generatividad también han sido descubiertos en muestras de diferente procedencia cultural 
FIGURA 3

Modelo de la generatividad (McAdams, Hart y Maruna, 1998)
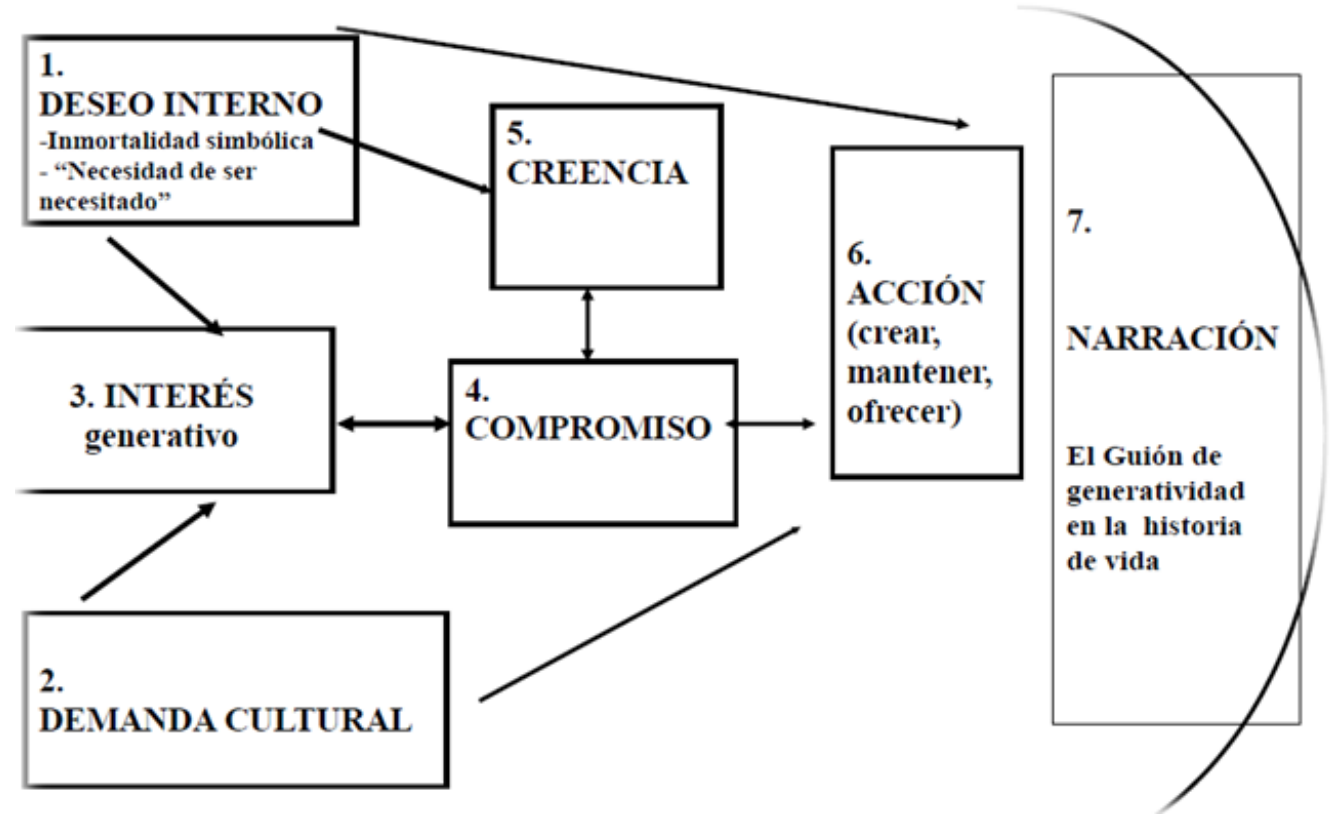

como Alemania, Camerún y la República Checa (Bush, Hofer, Solcova y Tavel, 2018) o en diferentes grupos étnicos (Armstrong, 2003).

A la luz de los hechos descritos, parece lícito afirmar que la cultura en sí misma no afecta a los mecanismos psicológicos básicos de la generatividad. Podría decirse que existe una generatividad psicológica en todas las culturas. Esta afirmación reduce el papel o efecto de la cultura en la generatividad a priori, sin embargo, es posible que su efecto sea observable en el plano más expresivo y tangible. Un estudio con participantes norteamericanos preguntó a los sujetos cuáles creían que eran los componentes o esferas de la generatividad. Los elementos nombrados por los sujetos no correspondían con los del modelo de la generatividad (Figura 3). Rubinstein, Girling, De Medeiros, Brazda y Hannum (2015) argumentaron que los conceptos del modelo eran demasiado abstractos para representar razones o esquemas generativos culturales popularmente. Esto podría suponer que los elementos del modelo eran demasiado psicológicos como para mostrar diferencias culturales. Si la premisa es que la cultura afectará a la concepción de la generatividad, la diferencia transcultural será visible entonces en el comportamiento y expresión generativa, es decir, en las ideas, pensamientos y acciones que las personas toman como generativas. 


\section{Efectos e interpretaciones culturales de la generatividad}

Si nos remontamos al origen del concepto generatividad, ya algunos autores (Hoare, 2000; Hulsizer et al., 1981) reprocharon a Erikson que las fases del desarrollo psicosocial que describió estaban construidas bajo la influencia de la cultura occidental. Busch y Hofer (2011), por su parte, expresaban que existía necesariamente una diferencia en la forma en que las culturas construyen el comportamiento prosocial, aunque fuera mínima. En un estudio con gemelos (Fabbender, Wiebe y Bates, 2019), en el que se pretendía por primera vez distinguir la parte genética y ambiental de la generatividad, se concluyó que los ambientes no compartidos entre los gemelos tenían un efecto significativo. Este hallazgo, en efecto, va en la línea de que la exposición a normas y sistemas culturales diversos juega un rol esencial en la concepción y expresión de la generatividad.

Esta y otras demostraciones de la existencia de un componente cultural que afecta a la generatividad han llevado a algunos autores a hipotetizar a la luz de sus resultados diferencias en la interpretación del comportamiento generativo a partir de la cultura. Según Doty (1994), en culturas individualistas como las occidentales, el miedo a la muerte, a dejar de existir individualmente, podría dar lugar al comportamiento generativo. En el caso de culturas orientales más colectivistas, en las que la importancia del individuo es significativamente menor, valores como transmisión y preservación de la cultura podrían ser el motor generativo. Hofer et al. (2008) proponen que en las sociedades colectivistas la expectativa es la de involucrarse en el cuidado y educación de las personas incluso aunque no sean parientes. De algún modo, las personas que viven en este contexto cultural estarían psicológicamente preparadas para este comportamiento y mostrarían un interés generativo superior. En contrapunto, en sociedades individualistas, la preocupación por el alcance de los objetivos y el desarrollo individual podría amenazar las oportunidades y el interés generativo. Estas tesis van en línea con los resultados de estudios en actitudes transculturales, como, por ejemplo, el de Janoff-Bulman y Leggat (2002), en el que se comprobaba que los miembros de culturas colectivistas valoraban con una actitud más positiva la obligación de ayudarse unos a otros. En respuesta a todos estos descubrimientos, Kotre (2004) advierte que, si bien algunas culturas favorecen y educan más en el comportamiento prosocial y generativo que otras, ninguna cultura es eminente o permanentemente generativa, ya que los generativos son sus miembros individuales cuando llegan a una edad relativamente avanzada. Aquí Kotre hace referencia a que, según Erikson, la generatividad aparecía a partir de la adultez media, con lo cual no podríamos decir que una cultura es generativa, ya que muchos de sus miembros no habrían alcanzado el conflicto generatividad-estancamiento descrito 
por Erikson. Podría proponerse, sin embargo, que existen culturas que favorecen el interés y el comportamiento generativo.

Algunos de los resultados de los estudios de esta revisión siembran ciertas dudas a la hora de la generalización cultural de sus resultados. Existen estudios que hablan de la relación entre el comportamiento generativo y el respeto percibido en los adolescentes (Tabuchi, Nakagawa, Miura y Gondo, 2008; Cheng, 2009). Esta relación ha sido observada en países de cultura asiática. Son los propios autores de estos estudios los que dudan seriamente de que los valores y las normas culturales de países y sociedades occidentales dieran lugar a los mismos resultados. En su estudio, Timilsina, Kotani y Kamijo (2019) hacen referencia a una crisis generativa. En este trabajo los sujetos de sociedades rurales muestran niveles significativamente más altos de prosocialidad y generatividad que las sociedades urbanas. Los autores argumentan que las sociedades más competitivas, social y económicamente, presentan cambios culturales que suponen una amenaza para el interés generativo. Se avanza en este sentido hacia la formación de nuevas culturas donde la generatividad puede no tener cabida. Debemos asumir en este punto que los cambios culturales también suponen cambios en la generatividad de las personas.

\section{Evidencias culturales de la aplicación y utilidad potencial de la generatividad}

Es interesante, para finalizar, ver que las diferencias culturales en cuanto a generatividad no se limitan a los ámbitos antes citados en este texto, sino que también se encuentran diferencias en la utilidad que las diferentes culturas dan al comportamiento generativo. En culturas indígenas de Alaska (Lewis y Allen, 2017) el comportamiento generativo constituye una motivación y un factor de protección respecto a adicciones de diferentes tipos. En ese sentido, la generatividad no solo ayuda al propio sujeto y los integrantes más jóvenes de la comunidad, sino que es un elemento social que tiene efectos positivos en la comunidad en general (alejando a personas de comportamientos de abuso o adicción a sustancias). En Norteamérica, el comportamiento generativo sirve también para combatir el aislamiento y el estigma de enfermos mentales (Milstein, Middel y Espinosa, 2017). También se involucra a las personas con más edad en planes de preparación para catástrofes en las que tienen un papel importante para la comunidad. De esta manera, las personas con más edad se sienten necesarias y parte de la comunidad y el trabajo que ejercen redunda en un beneficio comunitario (Ashida, Robinson, Gay y Ramirez, 2016). En países pertenecientes a otras culturas, como India o Cuba, se pide asesoramiento a personas ancianas acerca de desastres naturales y planes de emer- 
gencia (WHO, 2008). Estos ejemplos no son más que otra evidencia de que la cultura está involucrada en múltiples facetas de la generatividad.

\section{DISCUSIÓN}

En esta revisión sistemática se ha examinado la relación entre la cultura y la generatividad con base en los trabajos empíricos y teóricos de los últimos treinta años. En cuanto a las variables más psicológicas que conforman la estructura de la generatividad, podemos afirmar que son comunes entre las culturas. El papel de la cultura no está en ese lugar. El modelo de generatividad de McAdams y De St. Aubin (1992) ha sido probado con éxito en culturas realmente dispares. Sin embargo, en cuanto a la concepción, los motivos y la educación en el interés y el comportamiento generativo, las diferencias socioculturales emergen irremediablemente. Si bien la generatividad es un concepto que podemos encontrar a nivel individual en todas las persones (con mayor o menor nivel), la cultura en la que se vive y actúa juega un papel fundamental en la educación, los motivos y la evolución de la generatividad, sobre todo en su ámbito más sociológico.

En las culturas más individualistas, la fiebre de la consecución de objetivos y la evolución egoísta y distante intergeneracionalmente amenazan seriamente el comportamiento generativo. Son las culturas colectivistas las que, en este ámbito, continúan preservando y alimentando la fuente generativa más inacabable, la protección-trasmisión de la cultura colectiva. De estas conclusiones puede extraerse que la cultura es una base y un marco para la generatividad, que provoca cambios en todo el proceso generativo y que, sin cultura, sería difícilmente entendible la generatividad para cualquiera de nosotros. Esta visión acerca de la relación entre estos dos conceptos es mucho más amplia y ambiciosa que la concepción de la relación como un tipo de generatividad: generatividad cultural. Si bien ambas visiones no son incompatibles, no debemos olvidar que el estudio de la cultura en la generatividad no puede limitarse a la trasmisión de valores y normas culturales entre generaciones.

Esta revisión también reivindica la importancia de la cultura en el estudio del ámbito generativo y proporciona una base clara y sólida para futuras investigaciones empíricas, que deben perseguir no tanto los constructos psicológicos generativos en diferentes culturas, sino cómo la cultura influye en lo que las personas piensan y sienten como generatividad. No debemos olvidar que el comportamiento generativo puede ser también una herramienta para buscar el beneficio de todos y la evolución sin la pérdida de costumbres, historia y valores. Debe tenerse en cuenta que una limitación en este estudio ha sido el número considerable de artículos que presentaban un análisis cualitativo 
de la información recabada en entrevistas. Aunque se ha tenido suma precaución y objetividad en las conclusiones expuestas en esta revisión, son necesarios más trabajos empíricos cuyo tema central sea la relación entre generatividad y cultura.

En la línea de lo expresando por Kotre (1994) a lo largo de su estudio en la generatividad, no existe forma de sobrevivirse a uno mismo, de ser generativo, sin el vehículo de la cultura.

\section{BIBLIOGRAFÍA}

Alexander, B.B., Rubinstein, R.L., Goodman, M., y Luborsky, M. (1991). “Generativity in culture context: The self, death and immortality as experienced by older Americanwomen".AgeingandSociety, 11(4):417-442.DOI: 10.1017/S0144686X00004384

Armstrong, M.J. (2003). "Is being a grandmother being old? Cross-ethnic perspectives from New Zealand”. Journal of Cross-Cultural Gerontology, 18(3): 185-202. DOI: 10.1023/B:JCCG.0000003089.53598.73

Ashida, S., Robinson, E.L., Gay, J., y Ramirez, M. (2016). "Motivating rural older residents to prepare for disasters: Moving beyond personal benefits". Ageing y Society, 36(10), 2117-2140. DOI: 10.1017/S0144686X15000914

Aydinli, A., Bender, M., Chasiotis, A., De Vijver, F.J.R.V., y Cemalcilar, Z. (2015). "Implicit and explicit prosocial motivation as antecedents of volunteering: The moderating role of parenthood". Personality and Individual Differences, 74: 127-132. DOI: 10.1016/j.paid.2014.10.011

Ball, J. (2010). «Indigenous fathers' involvement in reconstituting "circles of care"». American Journal of Community Psychology, 45(1): 124-138. DOI: 10.1007/ s10464-009-9293-1

Bates, J.S., y Goodsell, T.L. (2013). "Male kin relationships: Grandfathers, grandsons, and generativity". Marriage and Family Review, 49(1): 26-50. DOI: $10.1080 / 01494929.2012 .728555$

Berman, H.J. (1995). "Generativity and transference heroics". Journal of Aging Studies, 9(1): 5-11. DOI: 10.1016/0890-4065(95)90021-7

Berry, B. (2006). "What accounts for race and ethnic differences in parental financial transfers to adult children in the united states?". Journal of Family Issues, 27(11): 1583-1604. DOI: http://dx.doi.org/10.1177/0192513X06291498

Black, H.K., y Rubinstein, R.L. (2009). "The effect of suffering on generativity: Accounts of elderly African American men". Journals of Gerontology - Series B Psy- 
chological Sciences and Social Sciences, 64(2): 296-303. DOI: 10.1093/geronb/ gbn012

Busch, H., y Hofer, J. (2011). "Identity, prosocial behavior, and generative concern in German and Cameroonian Nso adolescents". Journal of Adolescence, 34(4): 629638. DOI: 10.1016/j.adolescence.2010.09.009

Busch, H., Hofer, J., Solcova, I.P., y Tavel, P. (2018). "Generativity affects fear of death through ego integrity in German, Czech, and Cameroonian older adults". Archives of Gerontology and Geriatrics, 77: 89-95. DOI: 10.1016/j.archger.2018.04.001

Cheng, S. (2009). "Generativity in later life: Perceived respect from younger generations as a determinant of goal disengagement and psychological well-being". The Journals of Gerontology: Series B: Psychological Sciences and Social Sciences, 64(1): 45-54. DOI: http://dx.doi.org/10.1093/geronb/gbn027

De Medeiros, K., Rubinstein, R., y Ermoshkina, P. (2013). «The role of relevancy and social suffering in "generativity" among older post-soviet women immigrants». Gerontologist, 55(4): 526-536. DOI: 10.1093/geront/gnt126

Doty, W.G. (1994). "Evolving beyond the adolescent warrior: Postheroic masculinist generativity". The Journal of Men's Studies, 2(4): 353-373. DOI: http://dx.doi. org/10.3149/jms.0204.353

Downey, H., Threlkeld, G., y Warburton, J. (2016). "How do older Australian farming couples construct generativity across the life course?: A narrative exploration". Journal of Aging Studies, 38: 57-69. DOI: 10.1016/j.jaging.2016.04.007

Ehlman, K., y Ligon, M. (2012). "The application of a generativity model for older adults". International Journal of Aging and Human Development, 74(4): 331-344. DOI: 10.2190/AG.74.4.d

Erikson, E.H. (1963). Childhood and society. Nueva York: Norton.

- (1982). The life cycle completed. Nueva York: Norton.

Faßbender, K., Wiebe, A., y Bates, T.C. (2019). "Physical and cultural inheritance enhance agency, but what are the origins of this concern to establish a legacy? A nationally-representative twin study of Erikson's concept of generativity". Behavior Genetics. DOI: http://dx.doi.org/10.1007/s10519-018-9943-x

Grünberg, K. (2007). "Contaminated generativity: Holocaust survivors and their children in Germany". The American Journal of Psychoanalysis, 67(1): 82-96. DOI: http://dx.doi.org/10.1057/palgrave.ajp.3350005

Hoare, C.H. (2002). Erikson on development in adulthood: New insights from the unpublished papers. Nueva York: Oxford University Press. 
Hofer, J., Busch, H., Au, A., Polackova Solcova, I., Tavel, P., y Wong, T.T. (2014). "For the benefit of others: Generativity and meaning in life in the elderly in four cultures". Psychology and Aging, 29(4): 764-775. DOI: 10.1037/a0037762

Hofer, J., Busch, H., Chasiotis, A., Kaertner, J., y Campos, D. (2008). "Concern for generativity and its relation to implicit pro-social power motivation, generative goals, and satisfaction with life: A cross-cultural investigation". Journal of Personality, 76(1): 1-30. DOI: 10.1111/j.1467-6494.2007.00478.x

Hofer, J., Busch, H., Chasiotis, A., Kärtner, J., y Campos, D. (2008). “Concern for generativity and its relation to implicit pro-social power motivation, generative goals, and satisfaction with life: A cross-cultural investigation". Journal of personality, 76(1): 1-30. DOI: 10.1111/j.1467-6494.2007.00478.x

Hulsizer, D., Murphy, M., Noam, G., Taylor, C., Erikson, E., y Erikson, J. (1981). “On generativity and identity: From a conversation with Erik and Joan Erikson". Harvard Educational Review, 51: 249-269.

Janoff-Bulman, R., y Leggatt, H.K. (2002). "Culture and social obligation: When "shoulds" are perceived as "wants"》». Journal of Research in Personality, 36(3): 260-270. https://doi.org/10.1006/jrpe.2001.2345

Kane, R. (1996). "From generation to generation: Thoughts on legacy". Generations, 20: 5-9.

Kotre, J. N. (1984). Outliving the self: Generativity and the interpretation of lives. Baltimore: Johns Hopkins University Press.

— (1995). "Generative outcome". Journal of Aging Studies, 9(1): 33-41. DOI: http:// dx.doi.org/10.1016/0890-4065(95)90024-1

- (1996). Outliving the self: How we live on in future generations. Nueva York: Norton.

- (2004). "Generativity and culture: What meaning can do". In E. de St. Aubin, D.P. McAdams, y T.C. Kim (eds.), The generative society: Caring for future generations (pp. 35-50). Washington, DC: American Psychological Society. https://doi. org/10.1037/10622-003

Krebs, A., Rieunier, S., y Urien, B. (2015). “Generativity: Its role, dimensions and impact on cultural organizations in France". International Journal of Arts Management, 17(3): 28-45.

Lewis, J.P., y Allen, J. (2017). "Alaska native elders in recovery: Linkages between indigenous cultural generativity and sobriety to promote successful aging". Journal of Cross-Cultural Gerontology, 32(2): 209-222. DOI: 10.1007/s10823-017-9314-8

Makover, R.B. (2005). "Review of the generative society: Caring for future generations". The American Journal of Psychiatry, 162(1): 199. DOI: http://dx.doi. org/10.1176/appi.ajp.162.1.199 
Manheimer, R.J. (1995). "Redeeming the aging self: John Kotre, George Drury, and cultural generativity". Journal of Aging Studies, 9(1): 13-20. https://doi. org/10.1016/0890-4065(95)90022-5

McAdams, D.P. (2006). "The redemptive self: Generativity and the stories Americans live by". Research in human development, 3(2-3): 81-100. https://doi.org/10.1080/1 5427609.2006 .9683363

McAdams, D.P., y De St Aubin, E.D. (1992). "A theory of generativity and its assessment through self-report, behavioral acts, and narrative themes in autobiography". Journal of personality and social psychology, 62(6): 1003-1015. https://doi. org/10.1037/0022-3514.62.6.1003

McAdams, D.P., Diamond, A., De St. Aubin, E., y Mansfield, E. (1997). "Stories of commitment: The psychosocial construction of generative lives". Journal of Personality and Social Psychology, 72(3): 678-694. DOI: http://dx.doi.org/10.1037/00223514.72.3.678

McAdams, D.P., Hart, H.M., y Maruna, S. (1998). "The anatomy of generativity". En D. P. McAdams y E. de St. Aubin (eds.), Generativity and adult development (pp. 7-43). Washington, DC: American Psychological Association. https://doi. org/10.1037/10288-001

Milstein, G., Middel, D., y Espinosa, A. (2017). "Consumers, clergy, and clinicians in collaboration: Ongoing implementation and evaluation of a mental wellness program". American Journal of Psychiatric Rehabilitation, 20(1): 34-61. DOI: 10.1080/15487768.2016.1267052

Peterson, B.E., y Duncan, L.E. (1999). “Generative concern, political commitment, and charitable actions". Journal of Adult Development, 6: 105-118.

Robinson, O.C., Dunn, A., Nartova-Bochaver, S., Bochaver, K., Asadi, S., Khosravi, Z., Yang, Y. (2016). "Figures of admiration in emerging adulthood: A four-country study". Emerging Adulthood, 4(2): 82-91. DOI: 10.1177/2167696815601945

Rubinstein, R.L., Girling, L.M., de Medeiros, K., Brazda, M., y Hannum, S. (2015). "Extending the framework of generativity theory through research: A qualitative study”. Gerontologist, 55(4): 548-559. DOI: 10.1093/geront/gnu009

Tabuchi, M., Nakagawa, T., Miura, A., y Gondo, Y. (2015). "Generativity and interaction between the old and young: The role of perceived respect and perceived rejection". Gerontologist, 55(4) : 537-547. DOI: 10.1093/geront/gnt135

Timilsina, R.R., Kotani, K., y Kamijo, Y. (2019). "Generativity and social value orientation between rural and urban societies in a developing country". Futures, 105: 124-132. DOI: 10.1016/j.futures.2018.09.003 
Warburton, J., McLaughlin, D., y Pinsker, D. (2006). "Generative acts: Family and community involvement of older Australians". International Journal of Aging and Human Development, 63(2): 115-137. DOI: 10.2190/9TE3-T1G1-333V-3DT8

World Health Organization, WHO (2008). Older Persons in Emergencies: An Active Ageing Perspective. Recuperado de http://www.who.int/ageing/projects/ emergencies/en/ 